This item was submitted to Loughborough's Research Repository by the author.

Items in Figshare are protected by copyright, with all rights reserved, unless otherwise indicated.

\title{
Detecting impacts on a representative aerospace structure: an
} implementation with tests

\section{PLEASE CITE THE PUBLISHED VERSION}

http://dx.doi.org/10.12783/SHM2015/368

\section{PUBLISHER}

(C) DEStech Publications, Inc.

\section{VERSION}

AM (Accepted Manuscript)

\section{PUBLISHER STATEMENT}

This work is made available according to the conditions of the Creative Commons Attribution-NonCommercialNoDerivatives 4.0 International (CC BY-NC-ND 4.0) licence. Full details of this licence are available at: https://creativecommons.org/licenses/by-nc-nd/4.0/

\section{LICENCE}

CC BY-NC-ND 4.0

\section{REPOSITORY RECORD}

Bemment, Samuel D., lan Read, and Peter D. Hubbard. 2019. "Detecting Impacts on a Representative Aerospace Structure: An Implementation with Tests". figshare. https://hdl.handle.net/2134/18935. 
(FIRST PAGE OF ARTICLE) $^{1}$

\begin{abstract}
Collisions with aerial objects, e.g. bird strikes, pose a threat to aircraft in flight. In conventional aircraft, the pilot(s) would typically be aware of any significant collision through control response, noise, or visual indicators, and can fulfil the regulatory requirement of reporting the incident. In a UAV (Unmanned Aerial Vehicle), there is a requirement to automate these functions. The aim of the work detailed in this paper is to demonstrate that acoustic emission sensing equipment developed in the laboratory, and described in previous literature, can also be used to detect impacts on a large scale aerospace structure. The test structure for this work is a BAE Systems HERTI (High Endurance Rapid Technology Insertion) UAV. Simulated bird strike impacts are performed along the leading edge. Measurements are transmitted from sensors mounted on the wing to a processing system that deduces the location and energy of the impact by comparing the range of acoustic signatures. It is shown that the use of an array of 3 sensors enables repeatable detection and location of low energy impacts, demonstrating that acoustic detection of impacts is possible on a representative aerospace structure.
\end{abstract}

\title{
INTRODUCTION
}

Collisions with aerial objects pose a threat to aircraft in flight, with a large number of impacts attributed to bird strikes [1]. Bird impacts can vary from a glancing blow to a level of energy sufficient to cause significant structural damage. This damage can require the adaptation or curtailment of the mission to correspond to the

Samuel Bemment and Peter Hubbard of the School of Electrical, Electronic and Systems Engineering, Loughborough University, Loughborough, Leicestershire, LE11 3TU, United Kingdom. s.d.bemment@lboro.ac. Ian Read of BAe Systems PLC Advanced Technology Centre, Sowerby Building, PO Box 5, Filton, Bristol, BS34 7QW, United Kingdom 


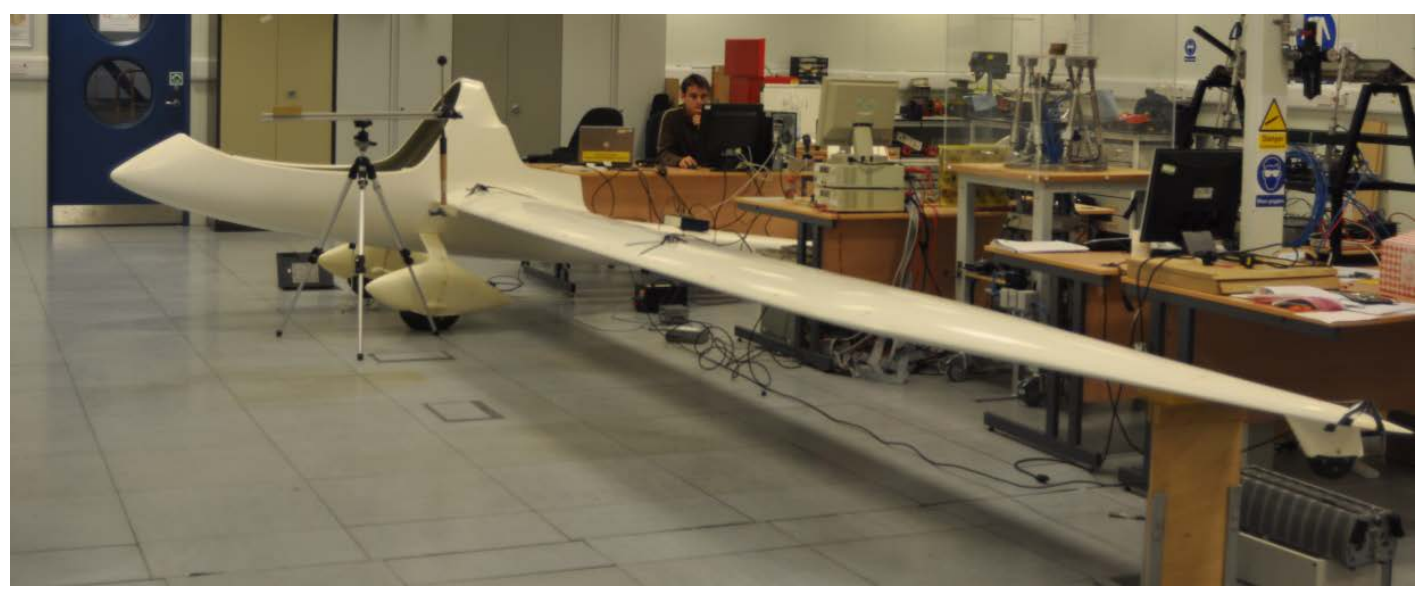

Figure 1. The HERTI Airframe in the laboratory.

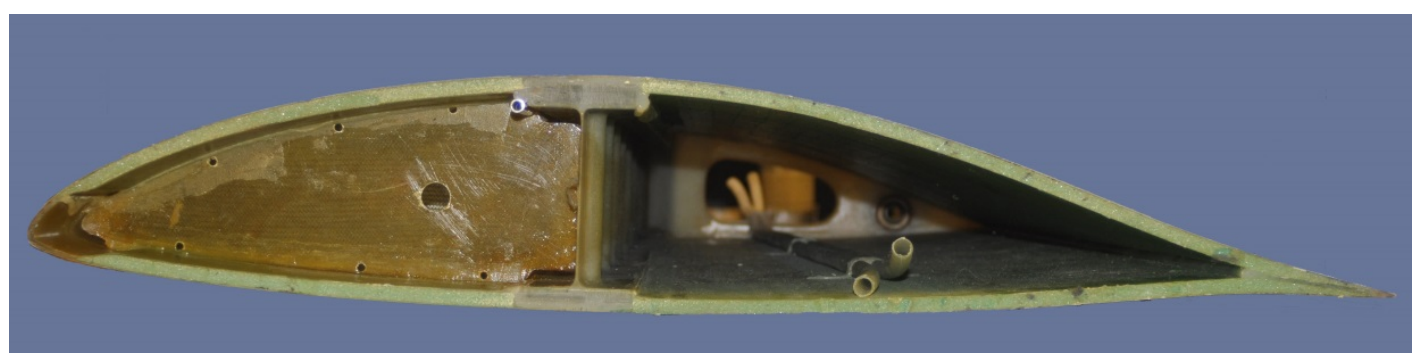

Figure 2. Cross-section of the HERTI Airframe wing structure, taken at 1240mm from wing root.

reduced performance of the aircraft. In a piloted aircraft, the pilot(s) would typically be aware of any significant bird strike through control response, noise, or visual indicators and can fulfil the regulatory requirement of reporting the incident [2]. The pilot can also make an assessment of the level of severity of the impact and decide whether to continue with the flight plan or take alternative action. In a UAV (Unmanned Aerial Vehicle), there is a requirement to automate these functions.

Previous work has addressed laboratory scale investigations into whether acoustic emissions sensors and sensor arrays, could reliably detect, locate and/or quantify the energy of impacts upon small $(1 \mathrm{~m} \times 2 \mathrm{~m})$ sheets of aerospacerepresentative material, with successful conclusions [3]. The aim of the work detailed in this paper is to demonstrate that the acoustic emission sensing equipment developed for these laboratory experiments can also be used to detect impacts on a large scale aerospace structure. The test structure for this work is the left wing of an airframe equivalent to the BAE Systems HERTI (High Endurance Rapid Technology Insertion) UAV [4], as shown in Fig. 1. The aim is to use the technology to detect and quantify impacts along the leading edge. In this experimental setup, the wing is supported at one end by the fuselage and at the other by a simple stand, and simulated bird strike impacts are performed along its span. Measurements are transmitted from sensors mounted on the wing to a processing system which deduces the location and energy of the impact by comparing the range of acoustic signatures. A graphical front end is included for demonstration purposes.

This paper presents the findings from the experiments conducted as part of this investigation. It is shown that the use of an array of 3 sensors has enabled repeatable 
detection and risk assessment of very low energy impacts ( 0.5J) along the wing span. The results obtained from these experiments show that acoustic detection for risk assessment of impacts is possible and can automatically provide basic information to satisfy the requirements to support decision making during missions, inform maintenance activities, and assist in regulatory reporting.

\section{Acoustic Sensing Concepts}

The idea of detecting impacts by utilising sensors embedded in a given structure is not new [5]. A range of approaches are available in literature. Prime examples include research performed into insulating foam strikes following the loss of the space shuttle Columbia [6], approaches involving the use of embedded optical fibres [7, 8], and approaches from the non-aerospace sectors, such as pipeline impact detection and monitoring [9]. These approaches have met with much success and now have a wide range of applications in structural health monitoring, extensively validated by experimental results $[10,11,12]$. Existing methods can be broadly sorted into two fields: fit-from-new, and retro-fit. Most methods require some element of modelling, or calibration, in order to achieve a level of accuracy/granularity suitable for application. Those which require minimal calibration are typically higher-cost, or more complex to integrate [5].

There now exists a segment of aircraft for which impact detection is, or will soon be, a requirement, which was not so some 10 years ago - UAVs. UAVs have, of course, existed for longer than this, but it is only recently rules and regulations for flying UAVs within civilian airspace, and amongst manned aircraft, have been drafted. A large fraction of the UAV population are small, relatively inexpensive aircraft, for example target drones, civilian or surveillance, and military (at the lower end of the cost and complexity spectrum, e.g. HERTI). It would not be justifiable to fit an expensive, complex or maintenance-intensive monitoring system to these aircraft. The requirements could be for a retro-fit or fit-from-new system, and a common system to satisfy both applications would be preferred. There is also the possibility of retrofitting a similar system to light aircraft in certain segments, as cost has historically been the preventing factor; though this is not explored further within this paper.

Previous proprietary work by BAE Systems has investigated the suitability of a network of COTS acoustic sensors to detect impacts, albeit on small, representative elements of aircraft structures. The sensors are linked to a processing unit which can discern impacts upon the structures. These type of sensors can be fitted into the structure at construction, or alternatively retro-fitted by simply bonding to the interior surface of panels through existing maintenance access panels. For this reason, the point-sensor approach may be preferable, from a cost and complexity viewpoint, to approaches involving embedded optical fibres, cited earlier.

This wing structure used herein is considerably more complex than those that have been examined previously in laboratory based experiments. It is also considerably larger. The increased size and complexity will affect the propagation of acoustic waves through the structure. It is these acoustic waves that the impact detection system uses to detect and locate impacts. The increased size means that acoustic waves can originate at greater distances from a sensor and consequently will suffer a larger attenuation. The increased complexity means that there may be multiple paths by which an acoustic wave can reach a sensor. This will be dependent 
on the origin of the acoustic wave and the location of the sensor. The aim of this work is to discern, through experimentation, whether the low-cost acoustic sensor network created in previous investigations can successfully locate, and discern the strength of, impacts upon a representative aerospace structure with such size and irregularities.

\section{TEST RIG}

The experimental test rig consists of three elements: the HERTI airframe, the bird strike simulation equipment, and the sensory data collection equipment. The software signal processing and visualization elements are considered separately below.

\section{The HERTI Airframe}

The HERTI is a UAV developed by BAE Systems PLC, which first flew in 2004 [4]. It is based upon a motor glider designed by J and AS Aero Design of Poland. The primary construction material is foam core GFRP (Glass Fibre Reinforced Plastic). A production prototype HERTI (previously been used for controller and actuation prototyping) is located at Loughborough University, with an in-tact and unmodified airframe. The left wing is supported at one end by the fuselage and at the other by a simple stand. Impacts can then be easily performed along its length. It was not practical to fit both wings to the fuselage, as the wing extends approximately $6 \mathrm{~m}$ from the wall of the fuselage. Figure 1 shows the HERTI airframe in the laboratory, including the simple stand under the wingtip in the foreground.

Figure 2 shows a cross-section of the wing structure, located $1240 \mathrm{~mm}$ from the wing root. At this point (and for the majority of it length) the chord is approximately $800 \mathrm{~mm}$. Much of the wing skin employs a foam core. The only exceptions are the leading edge and the region where the spar contacts the skin; here the skin is monolithic. At the spar the wing has a vertical dimension of $187 \mathrm{~mm}$; the web of the spar is $2 \mathrm{~mm}$ thick and contains vertically orientated, semi-circular ridges to improve its stiffness. These ridges are arranged periodically along the length of the spar with a spacing of approximately $107 \mathrm{~mm}$. Forward of the spar there are a series of baffles. These appear to be distributed along the wing with a spacing of approximately $220 \mathrm{~mm}$. The wing also contains a control surface and an air brake, and tapers towards the tip, further complicating its structure; these features are further detailed in Figure 4.

\section{Bird strike Simulator}

The Bird Strike simulator is a simple, manually operated arrangement capable of generating impacts up to 9J. It consists of a tripod stand, offering a method of adjusting the height and distance along the wing of the impact. The impact itself is provided by a mass upon a lever arm around a pivot. The mass has a substantial static counterweight to prevent the motion affecting the platform. The mass has a striking point which is covered with several layers of foam rubber to fully transfer impact energy. To simulate a strike, the mass is pivoted back from the wing, to an angle corresponding to the approximate required impact energy. This angle is defined by Equation 1, which provides a general release angle $\theta$ for a required impact energy $E$. 


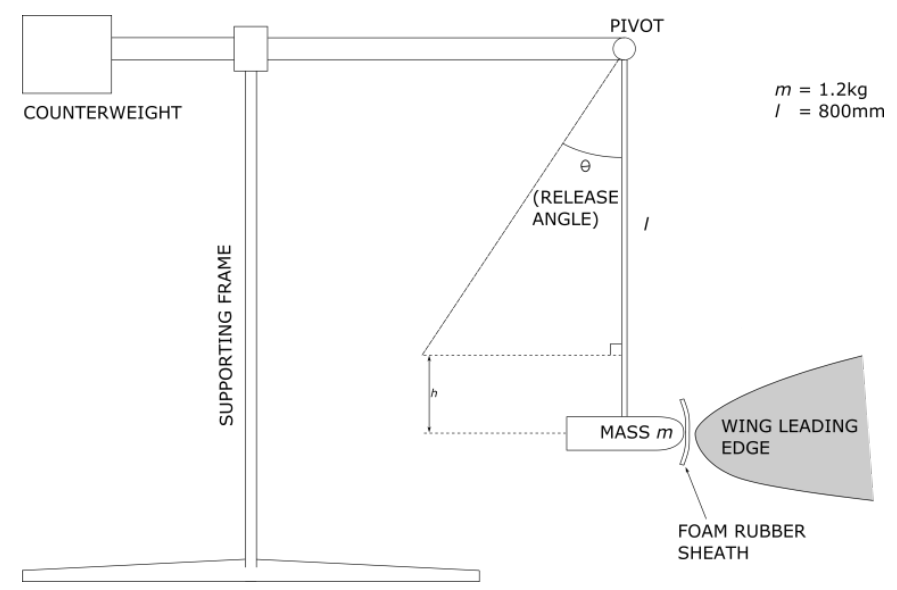

Figure 3. Bird strike simulation equipment, showing values for $m$ and $l$ in this instance

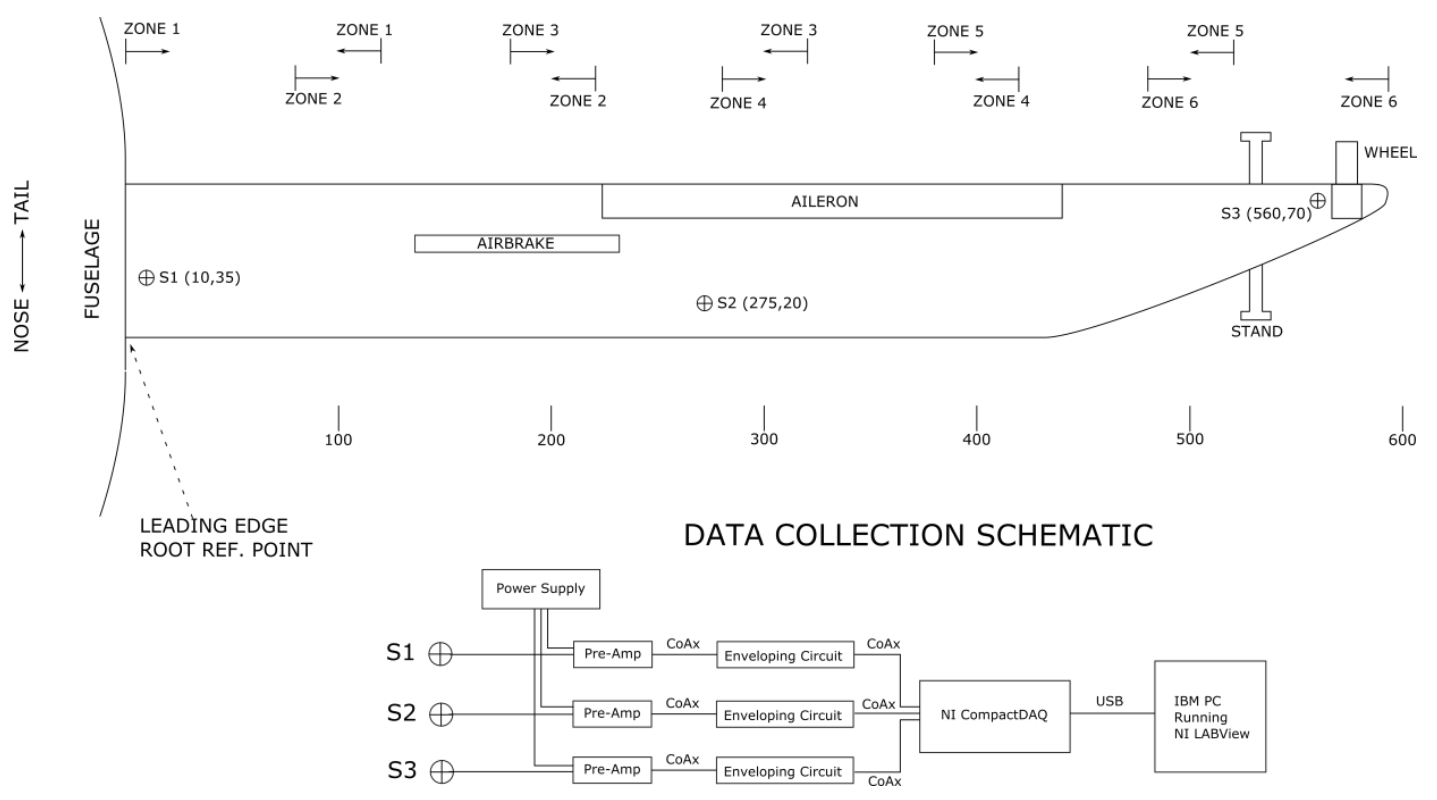

Figure 4. Dimensions and general layout of wing, and data collection schematic.

The mass is released by hand, and allowed to impact the wing a single time before being arrested. Figure 3 shows further details of this arrangement.

$$
\theta=\cos ^{-1}\left(\frac{l-\frac{E}{m g}}{l}\right)
$$

\section{Data Collection Equipment}

The data collection equipment used for this experiment is a set of commercial acoustic emissions sensors mated via custom pre-amp/enveloping hardware to NI (National Instruments) CompactDAQ I/O linked via USB to a PC running NI LabVIEW. The LabVIEW code consisted of a cycling buffer, set to record values 
when any sensor channel crossed a given threshold, with given pre-trigger. This final arrangement is shown in Figure 4.

The sensors (Physical Acoustics, 'WDI' series) operate at high frequency (100 kHz to $1 \mathrm{MHz}$ ) and have good low frequency discrimination; they are therefore less susceptible to low frequency background noise, e.g. from engines. The sensors were attached to the outside top wing skin with an acoustic bonding paste and adhesive. This location was selected so as to facilitate position adjustment, in a flight application it is anticipated they could be mounted internally. The signals from the sensors were captured using the prototype acoustic emission sensing system developed in previous work.

\section{SIGNAL PROCESSING}

Initial calibration activities used $1 \mathrm{~J}$ impacts sampled from two sensors only, in order to gauge the signal amplitude, SNR (Signal to Noise Ratio), and differential TTA (Time To Arrival) of the leading edge of the impact signal. This work revealed that whilst the sensors were suitable for application, the differential TTA of the signal at sensors more than $\sim 4 \mathrm{~m}$ apart was sufficient that arrival data would be clipped by the data acquisition system running at high frequency $(>500 \mathrm{kHz})$ and with a limited buffer space. However, the main signal features required by an impact detection system are:

- The time-of-arrival of the leading edge of the impact signal which can be used to estimate the location of the impact; and

- The signal energy which can be used alongside location information to determine the impact energy.

Therefore, while the high frequency response of the sensors is useful to eliminate background noise, the frequency make-up of the signal is not an essential feature for an impact detection system. The solution was to apply a hardware signal processing element before the DAQ (Data AQuisition System), such that the sample rate of the DAQ could be lowered without sacrificing necessary signal information, meaning the effective time span of the cycling buffer was elongated. This signal processing element consisted of an amplifier, enveloping element, and low-pass filter with cut-off at $30 \mathrm{kHz}$. The alternative was a substantial upgrade to the sampling equipment, but this was considerably more expensive. Figure 5 shows the effect of this circuit in reducing high frequency content and enveloping the waveform.

\section{FINDINGS}

\section{Discerning Impact Location}

In previous work where the impact material consisted of a uniform section of homogenous aerospace representative material, the speed of sound in that material could be established and impact locations triangulated accordingly. In a representative structure, there are various materials bonded together, where each material has a particular speed of transmission, and each structure shape has a particular ability to direct soundwaves. This structure leads to an attenuation of the shockwave from the 


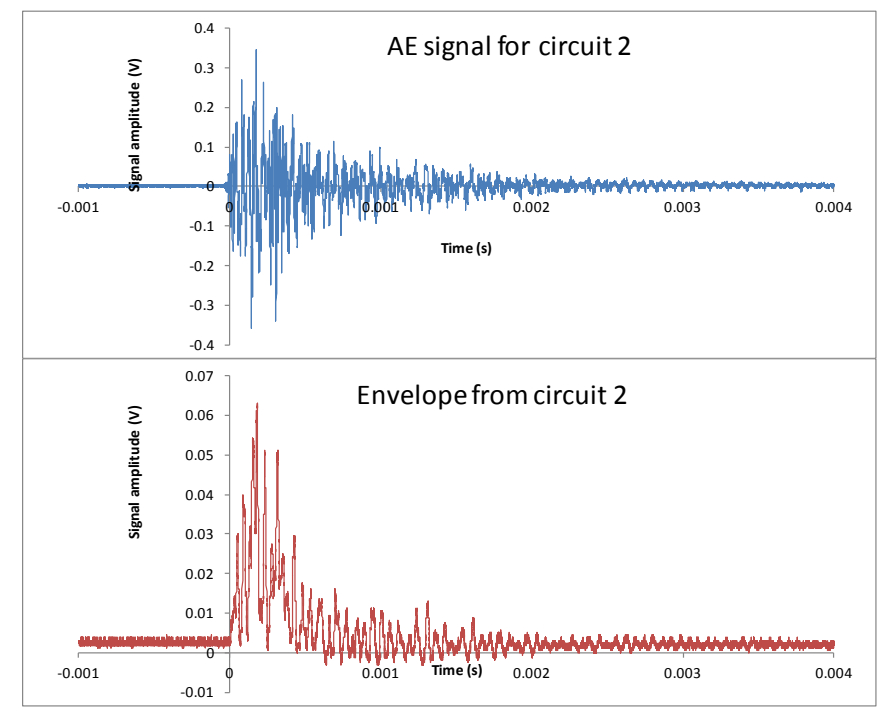

Figure 5. Raw sampled acoustic sensor data from a 1J impact (top) and output from the enveloping and low-pass filter (bottom) for an impact adjacent to sensor 2.

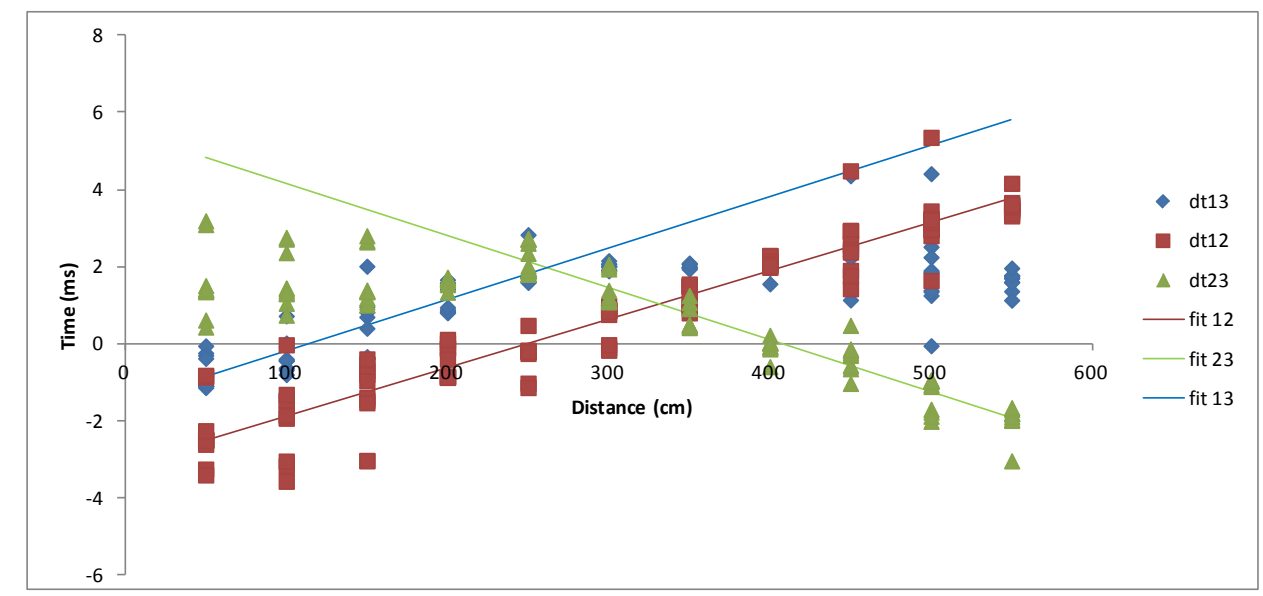

Figure 6. Difference in time of wavefront arrival (ms) vs distance from wing root $(\mathrm{cm})$, for a range of impact locations upon leading edge, for a single density of foam rubber.

impact over distance, alongside potentially shifted arrival times. It was postulated that factors contributing to location could be established experimentally through observing trends in the measurements. Three different densities of foam rubber sheath were used on the impact face for 6 impacts each of $2 \mathrm{~J}$ conducted at $500 \mathrm{~mm}$ intervals from the root of the leading edge. The three measured variables were the difference in time of arrival of the wavefront, established by thresholding, at the three sensors, labelled $d t_{12}$, $d t_{13}$ and $d t_{23}$, respectively. Figure 6 shows the results for one type of rubber sheath, which also includes linear fits using the least squares method. There is considerable scatter in this data, and further experimentation showed that achieving an accurate position fix purely by triangulation would be difficult as a result of this.

An alternative was proposed in which the wing was separated into impact zones, broadly corresponding to features upon the wing, and overlapping to allow for scatter. A logic tree then derived which zone the impact was in, based upon a 
qualitative comparison of the three $d t$ variables. For instance, a comparison of $d t_{12}$ and $d t_{13}$ can be used to discern if an impact is on the inboard or outboard half of the wing. Various combinations of zones were trialled, and it was found that six overlapping zones, as shown in figure 4, offered reliable and repeatable detection location. In the event that the system logic cannot discern an impact location, it resorts to flagging an impact for 'any zone', meaning the whole wing. The zones are a reasonable compromise as they align with features upon the wing, such as control surfaces.

\section{Discerning Impact Energy}

It was further postulated that the severity of that impact could be discerned from the information content in the acoustic sensor signals. Several methods were evaluated, the most accurate and reliable being as follows. Once the location of impact had been deduced, the signal from the nearest sensor is taken in isolation, as this signal has typically suffered the least attenuation through the structure. The processor then calculates the mean enveloped signal magnitude over the first millisecond from the derived impact timestamp. Since an absolute figure for impact energy is not the goal only whether the impact is significant enough to indicate damage may have occurred the processing element can compare this signal to a reference magnitude, which specifies the lower energy bound. Much more reliable results were achieved when there was a unique reference magnitude for each sensor. The maximum distance of an impact from a sensor is of the order of $1.5 \mathrm{~m}$, and it was established that the attenuation over this distance was not enough to significantly detract from the results. Experiments were successfully conducted with $0.5 \mathrm{~J}, 1 \mathrm{~J}$ and $3 \mathrm{~J}$ boundaries, where the system proved its ability to correctly discern whether the energy is above or below threshold. Should the impact location system default to reporting an impact in 'any zone', the processor then analyses data from all three sensors, and reports the majority decision.

\section{SOFTWARE DEMONSTRATOR}

A software front-end has been created in LabVIEW for the purposes of demonstration of the detection ability of the system. The software features a diagrammatic representation of the aircraft, similar to that in Figure 4. When an impact is detected, highlights a given zone with an orange or red band to indicate lowor high- energy impacts respectively. The screen has been designed as a mock-up of what could be a health monitoring panel in a UAV control centre providing health information regarding many subsystems.

\section{CONCLUSIONS AND FUTURE WORK}

This paper has presented an implementation of a system designed to detect impacts upon the leading edge of a wing, in this case upon a BAE Systems HERTI aircraft. The system uses commercial off-the-shelf components and a custom enveloping circuit and can reliably detect impacts of $\sim 0.5 \mathrm{~J}$ energy, attributing them to 
a particular impact zone and indicating an estimate of that energy. A graphical front end has been developed for demonstration purposes.

Immediate further work will involve detecting impacts on other areas of the structure. Following this, sensors will be located internally before flight testing to ensure the approach is able to discern impact signals from background noise generated, for instance, by engines, airflow, and actuators.

\section{REFERENCES}

1. Civil Aviation Authority, United Kingdom. 2013. CAP772 Chapter 5: “Aerodrome Wildlife Strike Hazard Management and Reduction.”

2. Civil Aviation Authority United Kingdom. 2009. The Air Navigation Order 200, article 227. "Mandatory Reporting of Birdstrikes."

3. BAE Systems. December 2013. - Project 119968 "Assessment of impact sensing capability Phase 2."

4. Daly, M. 2010. “Jane’s Unmanned Aerial Vehicles and Targets”, Issue 34. HIS Global/Jane’s, Surrey, UK.

5. Staszewski, W., Boller, C., and Tomlinson, G. 2004. "Health Monitoring of Aerospace Structures: Smart sensor technologies and Signal Processing”, Wiley, Chichester, UK.

6. Prosser, B., Gorman, M., and Madaras, E. 2004. “Acoustic Emission Detection of Impact Damage on Space Shuttle Structures,” NASA.

7. Malki, A., et al. 1996. "Impact and vibration detection in composite materials by using intermodal interference in multimode optical fibres”, Journal of Applied Optics, 35, 5198-5201.

8. Green, A., et al. 1995. "Optical fiber sensing technique for impact detection and location in composites and metal specimens," Smart Materials and Structures, Vol.4 No. 2.

9. Allison, P. S., Chassaing, C. E., and Lethcoe, B. October 2009. "Acoustic impact detection and monitoring system”, US Patent No. 7,607,351.

10. Xu, Q. January 2014. "Impact Detection and Location for a plate structure using least squares support vector machines," Structural Health Monitoring, 13:pp5-18.

11. Ciampa, F., Meo, M. and Barbieri, E. November 2012, "Impact Localisation in composite structures of arbitrary cross-section,” Structural Health Monitoring 11: pp643-655.

12. Mujica, L. E., Vehi, J., Staszewski, W. and Worden, K. September 2008. "Impact Damage Detection in Aircraft Composites Using Knowledge Based Reasoning," Structural Health Monitoring, 7: pp215-230 